\title{
No statistically significant results for two removable orthodontic retainers
}

\author{
Abstracted from \\ Sun J, Yu YC, Liu MY, et al. \\ Survival time comparison between Hawley and clear overlay retainers: a randomized trial. \\ J Dent Res 2011; 90: 1197-2201. Epub 2011 Jul 19. PubMed PMID: 21771797. \\ Address for correspondence: W.L. Lai Department of Orthodontics, \\ State Key Laboratory of Oral Diseases, West China Hospital of Stomatology, \\ Sichuan University, Chengdu 610041, People's Republic of China. E-mail: wenlilai@hotmail.com
}

\section{Question: What are the survival times of Hawley and clear overlay orthodontic retainers?}

Design Randomised controlled trial.

Intervention Participants were randomly assigned to receive either a Hawley retainer (HR) or a clear overlay retainer (COR). The HR was composed of a $2 \mathrm{~mm}$-thick acrylic resin base plate, one-arm clasps with $0.9 \mathrm{~mm}$-diameter stainless steel wire on the first molars, and a Hawley bow with $0.7 \mathrm{~mm}$ stainless steel wire. The COR was made from a 0.75 mm-thick thermoplastic material with the buccal edge paralleling the gingival margin, the lingual portion extending 4 to $5 \mathrm{~mm}$ beyond the lingual gingival margin. Distally the second molars were covered. Both groups of patients were required to wear the retainers full-time, except during meals and were followed for one year.

Outcome measure Retainer survival was the primary outcome.

Small surface cracks were not considered as a breakage unless the retainers could not be worn because of crack expansion.

Results One hundred and twenty patients were randomised, 61 in the HR group, 59 in the COR group, nine patients (five in the HR group and four in the COR group) were lost to follow up. Fracture was the most often cited reason for breakage, followed by retainer loss. No significant differences were seen in survival times between the two groups. Both retainers tended to fracture, but the fracture locations were different. Conclusions This analysis suggests that the survival times of the HRs and CORs were not statistically significantly different. Therefore, clinicians could advise their patients to wear either of the two retainers without taking breakage into consideration.

\section{Commentary}

Retention be it fixed or removable, is an important phase for orthodontic patients in order to maintain the position of the teeth after treatment is completed. It is advisable for almost all treated malocclusions. Removable retainers may be indicated for shorter periods of time as opposed to fixed retainers. Some practitioners recommend retention for longer times depending on individual patient factors. However a 2003 Cochrane review highlighted that there is not enough evidence to recommend one technique over another. ${ }^{1}$

Patient compliance and durability of the appliance are important consideration in selecting the right material and techniques. Another important factor is of cost for both practitioners and their patients.
If retention is recommended the materials selected are expected to be sufficiently strong to avoid breakage and continuous replacement. This randomised clinical trial had a well-formulated clinical question to evaluate the difference in fracture between the two commonly used techniques for retention. A flow chart was presented following the CONSORT approach (http://www.consort-statement. org/consort-statement/flow-diagram0/) to demonstrate and assess the progress of the trial.

The randomisation was appropriately done by a computerised system and allocation concealment was done to avoid selection bias with both groups being treated equally.

The drop out rate was minimal (less than 10\%) and equivalent in both groups.

The results showed no statistically significant differences between the two techniques.

However, the authors reported in the discussion section that no power calculation was done to determine the number of participants needed in each arm to affect the results and provide statistically significant calculations. A new trial with more participants would be necessary to precisely determine the difference if it can be determined between both techniques.

In other studies, clear removable retainers seem to be an acceptable technique and relatively inexpensive compared to the Hawley retainer and more preferable to patients. ${ }^{2}$ Conversely, some studies favor the Hawley retainer for maintaining the posterior occlusion. ${ }^{3}$

Both types fracture but patients have more losses with the clear retainer. However the size of the trial does not result in stand-alone evidence to translate into practice; individual patient needs, costs and practitioner experience are needed to make the final decision.

\section{Practice points}

- Mandibular retainers in long-term use seem to fracture more often than maxillary retainers. Patients seem to lose clear retainers more often.

\section{Analia Veitz Keenan}

NYU College of Dentistry, New York, USA

1. Littlewood SI, Millett DT, Doubleday B, Bearn DR, Worthington HV. Retention procedures for stabilising tooth position after treatment with orthodontic braces. Cochrane Database Syst Rev. 2006 Jan 25; (1): CD002283. Review.

2. Hichens $\mathrm{L}$, Rowland $\mathrm{H}$, Williams $\mathrm{A}$, et al. Cost-effectiveness and patient satisfaction: Hawley and vacuum-formed retainers. Eur J Orthod 2007; 29: 372-378.

3. Sauget E, Covell DA, Boero RP, Lieber WS. Comparison of occlusal contacts with use of Hawley and clear overlay retainers. Angle Orthod 1997; 67: 223-230. 